\title{
Comparison of LASER and LED illumination for fiber-optic fringe projection
}

\author{
Steffen Matthias ${ }^{\mathrm{a}}$, Markus Kästner ${ }^{\mathrm{a}}$, and Eduard Reithmeier ${ }^{\mathrm{a}}$ \\ ${ }^{a}$ Institute of Measurement and Automatic Control, Leibniz Universität Hannover, Nienburger \\ Str. 17, 30167 Hannover, Germany
}

\begin{abstract}
The inspection of functional elements is a crucial part of modern production cycles. However, with higher integration of production machinery and products, the accessibility for measurement systems is more and more limited. A solution for this problem can be found in endoscopy techniques, which are able to transport the image information for optical measurement methods. In this paper, an optical inspection system based on the fringe projection profilometry technique is presented. The fiber-optic fringe projection system uses two highresolution image fibers to connect a compact sensor head to the pattern generation and camera unit. In order to keep inspection times low, the system is developed with particular focus on fast projection times. This can be achieved by using a digital micro-mirror device, which is capable of projecting grey-scale patterns at a rate of more than 10 images per second. However, due to the low numerical aperture of the optical fibers, a limiting factor for the pattern rate is the illumination path of the pattern generator. Two different designs of the illumination path are presented, which are based on a LASER light source as well as a LED light source. Due to low beam divergence and high intensities LASERs are well suited for fiber coupling. Unfortunately, the coherent property of the light has negative effects in certain measurement applications, as interference patterns, the so called speckle, appear on rough surfaces. Although speckle reducing methods are employed in the LASER beam path, the emergence of interference cannot be prevented completely. As an alternative, an illumination path based on a LED light source is demonstrated. To compare the effects of the speckle, based on measurements on a planar calibration standard both designs are compared in terms of phase noise, which is directly related to the noise in the reconstructed 3-D point data. Additionally, optical power measurements of both methods are compared to give an estimation of coupling efficiency. Finally, the capabilities of the system are shown based on measurements of a micro-contour standard.
\end{abstract}

Keywords: Fringe Projection, Image Fiber, LED, LASER, Speckle, Phase-Shift

\section{INTRODUCTION}

Fringe projection is a well established technique to acquire areal height information in short time. The basic design of a fringe projection system consists of a single camera and a single pattern projector arranged in a triangle with the specimen. In order to obtain a point cloud of the surface geometry, a sequence of predefined patterns is projected onto the surface and captured with the camera. By evaluating the deformation of the patterns in the camera image, the relative 3-D position of surface points can be triangulated. While measuring large geometries is unproblematic due to the scalability of a fringe projection setup, the inspection of small elements on large carrier geometries pose a challenge as a result of shadowing. A number of surface points may be illuminated by the projector part, but not be visible in the camera part due to obstruction by the specimen's geometry. The resulting point cloud may therefore miss required data for the inspection of certain elements.

This problem is especially critical with complex large machinery for manufacturing filigree parts. An example is the new sheet bulk metal forming process, which is being researched within the collaborative research center SFB/TR 73 (www.tr73.de) funded by the German Research foundation (DFG). The aim of the new forming process is to manufacture deep-drawing and bulk-forming elements on a single part in combined forming step. ${ }^{1}$

Further author information:

E-mail: steffen.matthias@imr.uni-hannover.de, Telephone: +49 5117624456

Optical Sensing and Detection IV, edited by Francis Berghmans, Anna G. Mignani, Proc. of SPIE Vol. 9899, $989905 \cdot$ C 2016 SPIE · CCC code: 0277-786X/16/\$18 · doi: 10.1117/12.2225601 


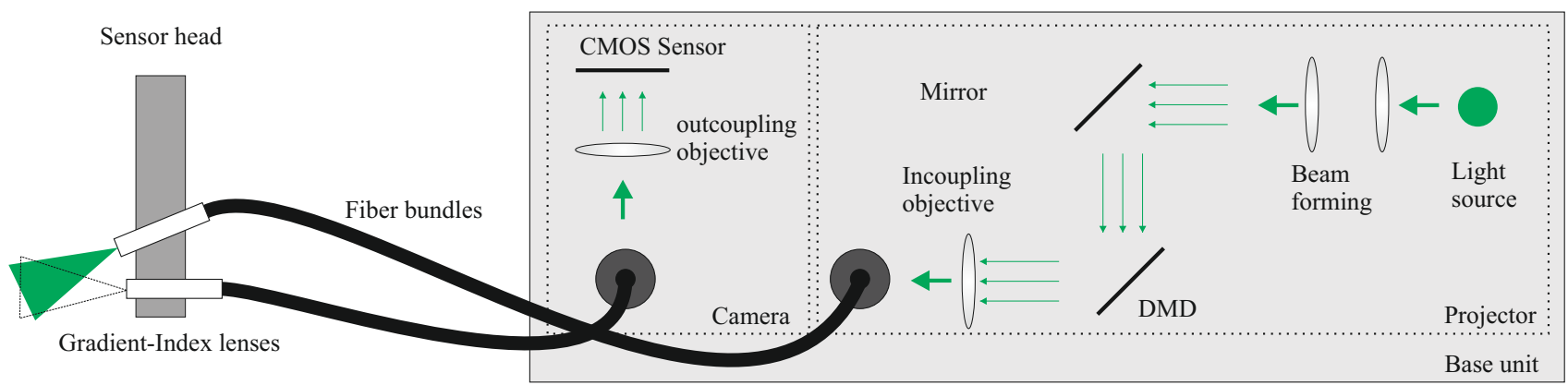

Figure 1. Schematic of the fiber-optic fringe projection system

One of the challenges in the research is the tool design, as some of the filigree tool parts are exposed to high forming forces. To avoid manufacturing defective parts due to defective tool geometries, periodic inspection of the forming tools in the running process is required. In order to fulfill this requirement, a new fiber-optic measuring system based on the fringe projection principle is being developed. The system enables the inspection of inner tool geometries by the use of a very compact sensor head, which is coupled to a base unit by high-resolution image fibers. Short measuring times are targeted as the forming machine needs to be halted during the inspection. To achieve low exposure times for the fringe patterns, the projector needs to deliver a high light output. A potential solution is the use of a laser light source, which allows for efficient fiber coupling of the generated fringe patterns. However, the coherent light leads to the emergence of speckle, which harms the pattern evaluation algorithms. Alternatively, LED light sources do not provoke the emergence of speckle, but are less efficient in fiber coupling.

After a short description of the fiber-optic fringe projection system, possible effects of speckle on the fringe pattern evaluation are discussed. A number of 2-D and 3-D measurements on different targets are shown with both LED and LASER illumination to give an estimation of the effects of speckle on measurements, followed by a discussion of the results.

\section{DESCRIPTION OF THE OPTICAL SYSTEM}

The fiber-optic fringe projection system consists of a base-unit, which houses the pattern generator and camera unit, and a compact sensor head. Both elements are coupled using two flexible high-resolution image fibers (100.000 pixels) with a diameter of $1.7 \mathrm{~mm}$ supplied by Fujikura Ltd. (type FIGH-100-1500N). A schematic of the system is shown in Fig. 1. The sensor head features gradient-index (GRIN) optics by GRINtech with diameter of $2 \mathrm{~mm}$ and a working distance of $10 \mathrm{~mm}$, resulting in a measuring volume of about $5 \mathrm{~mm} \times 5 \mathrm{~mm} \times$ $2.5 \mathrm{~mm}$. For larger measuring volumes GRIN optics with larger working distances are applicable.

The camera part of the base unit consists of a $4 \mathrm{x}$ microscope objective for fiber coupling and a Point Grey GS3U3-23S6M-C camera with a resolution of 1920 x 1200 pixels. For the projector part, two different light sources are available. A LED is employed as an incoherent light source with higher beam divergence in comparison with a LASER light source, while the latter suffers from speckle artifacts. The specifics of both illumination arrangements are described in the following paragraphs.

LED An OSRAM LV W5AM SMD LED is used for illumination because of its high optical flux at compact dimensions. High-power LEDs with larger active areas are impractical due to light loss at the small fiber aperture. The LED is specified by the manufacturer with a typical dominant wavelength of $505 \mathrm{~nm}$ at a typical optical flux of $67 \mathrm{~lm}$ at $350 \mathrm{~mA}$. In order to achieve even illumination of the DLP, the LED is used in a Köhler illumination setup.

LASER The LASER illumination setup features a Thorlabs DJ532-40 with a wavelength of $532 \mathrm{~nm}$ and a specified optical power of $40 \mathrm{~mW}$. The beam profile of the LASER diode is approximately Gaussian. Following the light source, the beam is targeted on a rotating diffuser plate in order to reduce speckle. ${ }^{2}$ The diffuser features a stochastic surface, which changes the beam's wavefront. Due to the rotating motion of the plate, the resulting interference patterns vary in time. Integrating the interference patterns over 
the camera's exposure time leads to a reduction of the speckle pattern's amplitude. A Köhler integrator, ${ }^{2}$ consisting of two micro-lens arrays with a lens pitch of $500 \mu \mathrm{m}$ by SUSS MicroOptics followed by a 150 $\mathrm{mm}$ Fourier lens, is used after the speckle reducer to homogenize the beam profile to a flat top. Due to the increased beam divergence introduced by the homogenizer arrangement, additional light is lost when the projector image is coupled to the image fiber, harming coupling efficiency and the homogeneity of the projection. However, it was observed that the overlap of brightness distributions induced by the beam homogenizer leads to an additional attenuation of speckle.

The active part of the projector is a digital light modulator DLP7000 from Texas Instruments. Individually tiltable micro-electro-mechanical mirrors on the surface of the device allow for the generation of arbitrary projection patterns with a resolution of 1024 by 768 pixels. Grayscale pattern sequences with a resolution of 8 bit can be displayed with a maximum pattern rate of $290 \mathrm{~Hz}$. The flexible generation of patterns is especially of use in the projection of object adapted inverse fringe projection patterns. ${ }^{3}$ To couple the generated images to the projecting image fiber, a $10 \mathrm{x}$ infinity corrected objective $(0.25 \mathrm{NA})$ is used in combination with a $100 \mathrm{~mm}$ tube lens, leading to a total de-magnification of the DLP image plane of approximately 5x. Both the DLP adjustment and fiber-coupling arrangement of the projector are identical for LED and LASER during the measurements.

\section{POTENTIAL EFFECTS OF SPECKLE ON FRINGE PROJECTION}

Speckle arises from illumination with coherent light sources, when parts of the beam's wave-front encounter different path lengths, for example due to optical elements in the beam path or the surface roughness of the illuminated object. The resulting interference of the illuminating light will lead to local changes in the amplitude of the reflected light. For projection applications, this results in multiplicative noise being applied to the desired projection pattern. In order to quantify the amount of speckle present in a camera image, the contrast of speckle patterns may be defined as the ratio of the standard deviation of intensity values to the average of intensity values. $^{4}$

$$
C=\frac{\sigma\left(I_{i}\right)}{\bar{I}_{i}}
$$

Common fringe projection systems use sinusoidal fringe patterns with four evenly chosen shifts with a combination of lower-frequency sinusoidal or encoded binary patterns for phase-unwrapping. ${ }^{5}$ The highest frequency pattern sequence usually carries the phase information which is employed in the calculation of 3-D data by triangulation. For a 4-pattern shift the per-pixel phase value may be calculated according to equation 2 .

$$
\phi=\operatorname{atan} 2\left(I_{1}-I_{3}, I_{2}-I_{4}\right)
$$

Constant spatial contrast variations, such as seen by variations in surface reflectivity or also stationary speckle patterns, are compensated in equation 2 by calculating the difference between patterns with a $180^{\circ}$ phase offset for both the real and imaginary component of the phase, removing constant image components. A stationary speckle pattern will therefore not directly impact the phase retrieval algorithm. However, high variations in local image contrast generally pose a challenge for the imaging device by requiring a high dynamic range.

A number of internal and external influences on the measuring device may lead to temporary changes in the beam pattern, which can not be compensated by the phase evaluation algorithm:

- Motion of the sensor head.

- Motion of the measuring object.

- Vibrations of the beam path.

- Modulation of the MEMS. 
While any relative motion of sensor head and measuring object should generally be avoided during the measuring process, already motion amplitudes of less than half of the light's wavelength, which are unavoidable in industrial conditions, will lead to significant changes in the speckle pattern. A different potential influence is the rotating diffuser, which induces time variations in the speckle pattern by purpose, which are supposed to average to a constant illumination over the camera's exposure time. In practice differences in the distribution will be present in different exposures. Additionally, the rotating motion of the plate and electrical motor introduces slight vibrations in the base unit. Finally, also displaying different patterns in the measuring process by modulating the micro-mirrors of the DLP will also modulate the speckle pattern.

\section{MEASUREMENTS}

In order to achieve comparable results, the adjustment of the fiber coupling objective and the DLP position has been kept constant for both light sources. Both illumination setups have been adjusted to fully illuminate the fiber coupled DLP area. As the output power of both LED and LASER illumination varies with temperature, measurements were performed after the optical output power was stable in an environment temperature of $20^{\circ} \mathrm{C}$. Table 1 shows measurements of the optical power of the projector after the image fibers and GRIN optics, obtained with a Thorlabs PW200 powermeter in combination with a S121C photo-diode sensor. Exact comparison of the efficiency of both sources is not possible based on these results, because the optical flux of the LED is specified with a tolerance of about $\pm 60 \%$ according to the datasheet. While the LED light source features a higher optical power for fringe projection, the coupling efficiency appears to be much lower than for the LASER source.

The images in Fig. 2 show detail views of a diffuse reflective point pattern calibration standard (Edmund Optics, dot spacing $0.125 \mathrm{~mm}$ ) through the camera fiber illuminated with the LASER and LED setup. Displayed next to the unfiltered cropped images a) and c) are the unfiltered DFT magnitude spectra b) and d) of the full camera image of the pattern. The spectrum of the LED illuminated pattern, displayed in d), features the fiber structure, which is visible as a faint ring. The dot pattern of the calibration standard can be seen on the inside of the ring with frequency components up to the Nyquist frequency of the image fiber, which is located approximately at half the diameter of the ring. In direct comparison, the camera image illuminated with the LASER light source is significantly more noisy, which is also visible in the magnitude spectrum. The bandwidth of the additional noise extends beyond the image fiber's Nyquist frequency. This indicates, that the speckle emerges from interference on the sensor's image plane. All images in the following analyses are low-pass filtered with a FIR-filter with the pass-edge located at the Nyquist-frequency of the image fibers. The filter therefore removes noise at frequencies that do not contain any image information. Especially the LASER setup benefits from the filter, as the higher frequency speckle noise is removed.

To estimate the temporary variance of the speckle effects, as well as the spatial variance, the micro fringe projection system is used to capture a sequence of 25 images of the diffuse reflective planar standard for both the laser and LED illumination. The cameras exposure time is set to $20 \mathrm{~ms}$ and the light output adjusted to be as high as possible without saturating the sensor. A region-of-interest covering 1677 pixels has been segmented in the center of the images to exclude reflectivity and vignetting effects in the evaluation. In order to estimate the spatial contrast of the speckle, the 25 images have been averaged and the contrast has been calculated according to equation 1. The temporal speckle contrast is calculated by spatially averaging over the 1677 pixel intensity values and also calculating the contrast of the resulting 25 intensity values according to equation 1 , giving an estimation of the modulation of the speckle pattern over time. For a third comparison parameter, also the amount of phase-noise present in the image is estimated by capturing 25 phase-maps in the same position as the camera image. The four step per frequency phase-shift as presented in section 3 is used with 40 fringe periods over the projector image for the highest frequency sequence. After applying the same region-of-interest as for the single-image evaluation, a line of best fit is calculated for each pixel column. As the phase-map is measured on a planar geometry and a region-of-interest with low lens distortion is selected, a line is a good approximation of

Table 1. Maximum optical power (white projector image)

\begin{tabular}{|l|l|}
\hline & Optical power \\
\hline LED & $5.8 \mathrm{~mW}$ \\
\hline LASER & $3.8 \mathrm{~mW}$ \\
\hline
\end{tabular}

Proc. of SPIE Vol. 9899 989905-4 


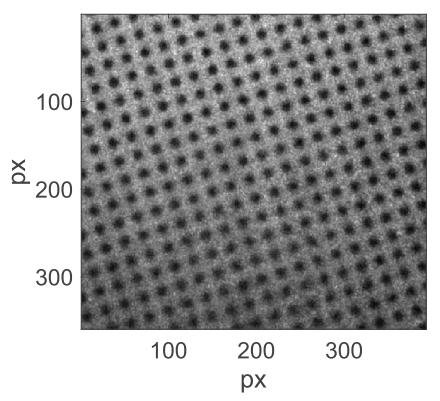

a) Camera Image (LASER)

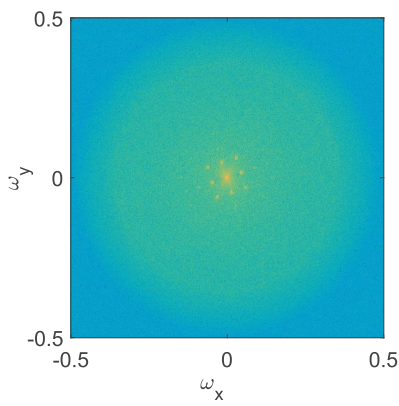

b) Magnitude spectrum (LASER)

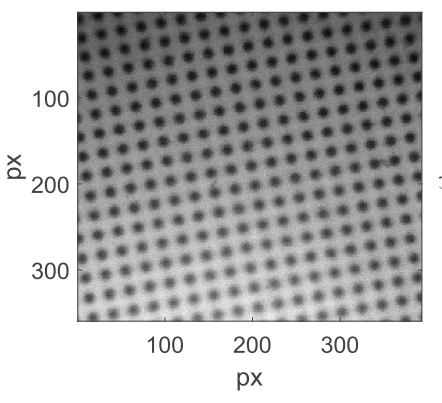

c) Camera Image (LED)

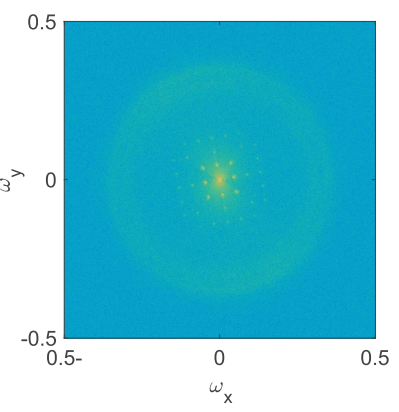

d) Magnitude spectrum (LED)

Figure 2. Detail views of camera images and corresponding magnitude spectra

Table 2. Comparison of temporary and spatial speckle effects

\begin{tabular}{|l|l|l|l|}
\hline & Temporal speckle contrast & Spatial speckle contrast & Phase noise \\
\hline LED & 0.00048 & 0.0211 & $0.0127 \mathrm{rad}$ \\
\hline LASER & 0.00830 & 0.0673 & $0.0319 \mathrm{rad}$ \\
\hline
\end{tabular}

the phase values in a camera column. The estimation of the phase noise is calculated by the standard deviation of the differences from the lines of best fit.

The results of the measurements are shown in table 2. Especially the temporal speckle contrast, which is critical for capturing phase-maps, is almost 20 times as high for the LASER as for the LED. Additionally, the spatial speckle contrast of the LASER illuminated plane is three times higher than on the LED illuminated measurements, which is visible in fig. 2 a) and c). Phase noise is $60 \%$ lower for the LED illumination.

In addition to the two-dimensional analysis of speckle effects, measurements on 3-D geometries are evaluated. The first measured geometry is the diffuse calibration standard, which has already been used in the 2-D evaluation. The second specimen is micro-contour standard (Alicona IF-Verification Tool), which features a number of cylindrical, step and trapezoidal geometries. While the calibration standard is mostly diffuse reflective, the surface of the micro-contour standard features higher specular reflection and surface roughness. For the following analysis the cylinder geometry with a radius of $1 \mathrm{~mm}$ is measured using the four step phase-shift technique with a maximum fringe frequency of 40 . Triangulation of the $3-\mathrm{D}$ point data is performed using a pinhole model for camera and projector after calibrating the model parameters. ${ }^{6}$ Both geometries have been captured in 25 measurements, for the planar target an area of 2104513 -D points has been segmented in each measurement, for the cylindrical target 77164 points per measurement. Using the Software GOM Inspect, a best fit plane and cylinder was fitted to each combined point cloud. Table 3 shows the standard deviation of the distances of the data points to the fitted reference geometries.

For the planar target, the 3-D points are scattered around the ideal geometry with a standard deviation of $2.5 \mu \mathrm{m}$ for the LED light source. The LASER illuminated plane exhibits more noise with a $92 \%$ higher standard deviation. On the cylindrical target, the standard deviation increases to $4.3 \mu \mathrm{m}$ for the LED light source, while the standard deviation of points measured with LASER illumination on the micro-contour standard is $165 \%$ higher.

Table 3. Standard deviations of point-surface distances

\begin{tabular}{|l|l|l|}
\hline & Diffuse planar object & Cylindrical object \\
\hline LED & $0.0025 \mathrm{~mm}$ & $0.0043 \mathrm{~mm}$ \\
\hline LASER & $0.0048 \mathrm{~mm}$ & $0.0114 \mathrm{~mm}$ \\
\hline
\end{tabular}




\section{DISCUSSION}

The influence of LASER speckle on the camera images is visible in figure 2 a) in comparison with the LED illuminated image in c). However, intensity variations are also present with LED illumination, as seen in the spatial speckle contrast measurement in table 2, as a result of the varying attenuation of the individual fibers in the fiber bundle and reflectivity variations on the standard. As explained in section 3, temporary variations of the speckle patterns, which superimpose the phase-shift patterns, have a much more critical influence on the phase reconstruction algorithm than static speckle patterns. The LASER exhibits a much higher temporal speckle variation, which results in more noise in the measured phase-maps.

When comparing the results of the 3-D measurements, both illumination setups show low deviations from the optically cooperative planar target. In this best-case situation the standard deviation is about twice as high for the LASER light source, indicating that robust reconstruction of phase-maps is possible despite temporal variations in speckle. However, for more a complex and optically less cooperative geometry, as presented with the cylindrical element on the micro-contour standard, the standard deviation increases notably more when using LASER illumination.

To conclude, the LED illumination exhibits significantly less noise in measured 3-D data especially for rough surfaces, while the LASER light source allows for efficient fiber coupling. As LEDs with higher radiant flux are currently only available with a larger active area and therefore not applicable for the fiber-coupled projector, LASERs are the only choice for reducing the measuring times of the fiber-optic fringe projection system. However, current measuring times of less than $3 \mathrm{~s}$ for typical geometries, achieved with LED illumination, are sufficient for the targeted application. Additionally, the LED avoids the necessity for laser safety precautions, such as the use of eye protection or specifically designed housings. To improve the results of the LASER based projector, more efficient speckle reduction techniques are available, for example by placing a static diffuser next to the moving diffuser. ${ }^{7}$ Vibrations in the optical path could be reduced with the use of a speckle reducer by Optotune, which applies motion to the diffuser by the use of electric polymers. ${ }^{8}$

\section{SUMMARY}

A new fiber-optic fringe projection system and its application in inspection tasks in modern production processes is presented. Two different projector setups based on LED and LASER illumination are introduced and both 2-D and 3-D measurements on objects with different optical and geometrical parameters presented. Significant more spatial noise is present in LASER illuminated camera images despite the use of speckle reduction techniques. Due to the compensation of static intensity modulations by the common four step phase shift algorithm, robust calculation of phase-maps is possible for both LASER and LED light sources. As temporary variations in the remaining speckle patterns cannot be avoided, the standard deviation of the distance of measured point clouds to reference objects was estimated to be at least twice as high for the LASER. For rough surfaces, such as seen in many technical applications, the effect of speckle is higher, leading to even more noise when measuring objects by LASER illumination. As the LED light source delivers sufficient optical power to achieve the measuring times of less than $3 \mathrm{~s}$ and avoids LASER safety issues, it is more suited for the targeted inspection tasks. However, if significant reductions in measuring times are required, the use of a LASER light source must be considered.

\section{ACKNOWLEDGMENTS}

The authors would like to thank the German Research Foundation (DFG) for funding the project B6 "Endoscopic geometry inspection" within the Collaborative Research Center (CRC) / TR 73.

\section{REFERENCES}

[1] Merklein, M., Allwood, J., Behrens, B.-A., Brosius, A., Hagenah, H., Kuzman, K., Mori, K., Tekkaya, A., and Weckenmann, A., "Bulk forming of sheet metal," CIRP Annals-Manufacturing Technology 61(2), 725-745 (2012).

[2] Voelkel, R. and Weible, K. J., "Laser beam homogenizing: limitations and constraints," Proc. SPIE $\mathbf{7 1 0 2 ,}$ 71020J-71020J-12 (2008). 
[3] Matthias, S., Ohrt, C., Pösch, A., Kästner, M., and Reithmeier, E., "Single image geometry inspection using inverse endoscopic fringe projection," ACTA IMEKO 4(2), 4-9 (2015).

[4] Goodman, J. W., "Some fundamental properties of speckle," J. Opt. Soc. Am. 66(11), 1145-1150 (1976).

[5] Peng, T., Gupta, S. K., and Lau, K., "Algorithms for constructing 3-d point clouds using multiple digital fringe projection patterns," Computer-Aided Design and Applications 2(6), 737-746 (2005).

[6] Matthias, S., Kästner, M., and Reithmeier, E., "Evaluation of system models for an endoscopic fringe projection system," Measurement 73, 239 - 246 (2015).

[7] Kubota, S. and Goodman, J. W., "Very efficient speckle contrast reduction realized by moving diffuser device," Applied Optics 49(23), 4385-4391 (2010).

[8] Blum, M., Büeler, M., Grätzel, C., Giger, J., and Aschwanden, M., "Optotune focus tunable lenses and laser speckle reduction based on electroactive polymers," Proc. SPIE 8252, 825207-825207-11 (2012). 